\author{
Professor Gabriela MIRCEA, PhD \\ Department of Business Information System \\ West University of Timişoara \\ E-mail:gabriela.mircea@e-uvt.ro \\ Professor Mihaela MUNTEAN, PhD \\ Department of Business Information System \\ West University of Timişoara \\ E-mail:mihaela.muntean@e-uvt.ro(Corresponding Author) \\ Professor Mihaela NEAMȚU, PhD \\ Department of Economics and Modelling \\ West University of Timişoara \\ E-mail:mihaela.neamtu@e-uvt.ro
}

\title{
ANALYSIS OF A CONGESTION MODEL IN A WIRELESS ACCESS NETWORK WITH ONE BOTTLENECK ROUTER AND N TCP FLOWS
}

\begin{abstract}
The paper analyses a model that describes a wireless access network with one bottleneck router and $n \geq 2$ TCP flows described by a nonlinear dynamical system. The equilibrium point is determined for the general case. For the particular case $n=2$ the periodic solutions are examined, when the round trip time is considered as bifurcation parameter. The conditions for the local asymptotic stability of the equilibrium point are given. In the last part, using Maple and Matlab, the numerical example verifies the theoretical results and some conclusions and future directions are shown.

Keywords: congestion, equilibrium point, local stability, Hopf bifurcation, wireless access network.

JEL classification: C61, C62, C63

\section{Introduction}

In the recent times, the wireless access network has paid great attention, because it is applied in different fields, especially to the Internet. The congestion control in wireless access network is important in the success of the wireless network technology [12]. According to [3], congestion control is an algorithm which allocates available resources to competing sources efficiently in order to avoid congestion collapse. The TCP congestion control algorithm has a role in avoiding
\end{abstract}


network flooding. Nowadays, TCP approximates the best capacity of the network by increasing/decreasing congestion window [5].

Therefore, there is a great motivation for many authors to study the congestion control algorithm. Also, the heterogeneous delays are taken into account in a wireless access network. In [3], the communication time delay is a bifurcating parameter and the behavior of the system is investigated. In [2]-[12], the local stability of the equilibrium point is studied in different congestion control models. Also, the Hopf bifurcation is examined when there is a single link and a single communication delay.

Based on the above papers, we conduct a study to find out the consequences of time delay in a wired access network with one bottleneck router and $n \geq 2 \mathrm{TCP}$ flows. The mathematical model is given by a nonlinear system with first order differential equations with time delay. For the general case we study the existence of the equilibrium point. When two different TCP flows pass through the router a detailed analyze is provided. When the round trip time is taken as bifurcation parameter, we apply the theory of differential equations with time delay to study the occurrence of the Hopf bifurcation and the periodicity of the orbits.

The structure of the paper is as follows. The mathematical models of the wired access network that takes into account the window size and the queue length of the router are displayed in Section 2. In Section 3 we prove that the mathematical model, with $n \geq 2$ TCP flows that pass through the router, has one positive equilibrium point. For this one the characteristic equation is written. The existence of Hopf bifurcation for the model with one bottleneck router and two TCP flows is analyzed in Section 4. A numerical example that illustrates the theoretical results can be found in Section 5. In the end, the conclusions are shown in Section 6.

\section{Mathematical models of a wired access network} by [5]:

The nonlinear differential equation that describes the window size is given

$$
\dot{w}_{i}(t)=x_{i}\left(t-\tau_{i}\right)\left(\frac{1-p_{i}(t)}{w_{i}(t)}-\frac{1}{2} p_{i}(t) w_{i}(t)\right), i=1, \ldots, n \text {. }
$$

where $w_{i}(t)$ is the TCP window size of flow $i, x_{i}(t)=\frac{w_{i}(t)}{\tau_{i}}$ represents the TCP rate, $\tau_{i}$ stands for the round trip time at time $t$ of flow $i$ and $p_{i}(t)$ is the probability of packet mark at time $t$.

There are two causes for the failure of a router in delivering packets: one can be if their data loads are corrupted, or the router buffers are already full. If $p_{d i}(t)$ denotes the drop probability, then (1) becomes: 
Analysis of a Congestion Model in a Wireless Access Network with One Bottleneck Router and N TCP Flows

$$
\dot{w}_{i}(t)=x_{i}\left(t-\tau_{i}\right)\left(\frac{1-p_{i}(t)}{w_{i}(t)}-\frac{1}{2}\left(1-p_{d i}\right) p_{i}(t) w_{i}(t)-p_{d i} p_{i}(t)\left(w_{i}(t)-1\right),\right), i=1, \ldots n
$$

The following differential equation describes the dynamics of the queue length [12]:

$$
\dot{q}(t)=F\left(\sum_{i=1}^{n} x_{i}\left(t-\tau_{i}\right)\right)-c, c>0
$$

where $c$ is the link capacity and $F$ is the adjusted rate of the source based on the congestion rate $x(t)$ from the link node. The nonnegative function $x(t)$ is a decreasing one and differentiable.

$$
\begin{aligned}
& \text { Since } p_{i}(t)=k q(t), x_{i}(t)=\frac{w_{i}(t)}{\tau_{i}},([6]), \text { equation (2) becomes: } \\
& \dot{x}_{i}(t)=x_{i}\left(t-\tau_{i}\right)\left[\frac{1-k q(t)}{\tau_{i}^{2} x_{i}(t)}-\frac{1}{2} k x_{i}(t) q(t)-\frac{1}{2} k p_{d i} x_{i}(t) q(t)+\frac{1}{\tau_{i}} p_{d i} q(t)\right] .
\end{aligned}
$$

In [12], the mathematical model for wireless access networks, with one bottleneck router and $n \geq 1$ identical TCP flows that pass through the router, is analyzed. In this case, for $x_{i}(t)=x(t), \tau_{i}=\tau, p_{d i}=p_{d}, i=1, \ldots, n, n \geq 1$, (4) becomes:

$$
\begin{aligned}
& \dot{x}(t)=x(t-\tau)\left[\frac{1-k q(t)}{\tau^{2} x_{i}(t)}-\frac{1}{2} k x(t) q(t)\left(1+p_{d}\right)+\frac{1}{\tau} k p_{d} q(t)\right], \\
& \dot{q}(t)=F\left(\sum_{i=1}^{n} x(t-\tau)\right)-c
\end{aligned}
$$

In this paper the wireless access network of only one bottleneck router and $n \geq 2$ different TCP flows passes though the router is considered. The mathematical model is given by:

$$
\begin{aligned}
& \dot{x}_{i}(t)=x_{i}(t-\tau)\left[\frac{1-k q(t)}{\tau^{2} x_{i}(t)}-\frac{1}{2} k x_{i}(t) q(t)\left(1+p_{d i}\right)+\frac{1}{\tau} k p_{d i} q(t)\right], i=1, \ldots, n, \\
& \dot{q}(t)=F\left(\sum_{i=1}^{n} x_{i}(t-\tau)\right)-c,
\end{aligned}
$$


Gabriela Mircea, Mihaela Muntean, Mihaela Neamtu

where $c>0$ and $F(x)$, and $x \geq 0$ is a decreasing and nonnegative derivative function.

\section{Analysis of system (6)}

The equilibrium point of system (6) is $\left(x_{1}^{*}, x_{2}^{*}, \ldots, x_{n 1}^{*}, q^{*}\right)$ and satisfies the system:

$$
\begin{aligned}
& \frac{1-k q}{\tau^{2} x_{i}}-\frac{1}{2} k x_{i} q(t)\left(1+p_{i}\right)+\frac{1}{\tau} k p_{i} q=0, i=1, \ldots, n, \\
& F\left(\sum_{i=1}^{n} x_{i}\right)-c=0
\end{aligned}
$$

From (7) we have:

$$
q=\frac{2}{k\left(2+\left(1+p_{i}\right) \tau^{2} x_{i}^{2}-2 p_{i} \tau x_{i}\right)}, i=1, \ldots n
$$

and $0<k q \leq 1$.

Hence $\left.\left(1+p_{i}\right) \tau^{2} x_{i}^{2}-2 p_{i} \tau x_{i}\right)$ and we get:

$$
H_{1}: \quad \tau \geq \frac{2 p_{i}}{\left(1+p_{i}\right) x}, i=1, \ldots, n
$$

We also consider:

$$
H_{2}: p_{1}>p_{i}, i=2, \ldots, n
$$

In what follows, we take $\tau \in\left[\tau_{1}, \infty\right)$, where

$$
\tau_{1}=\frac{2 p_{1}}{x_{1}\left(1+p_{1}\right)}
$$

With (7) and (9) we obtain:

$$
\tau\left(1+p_{1}\right) x_{1}^{2}-2 p_{1} x_{1}=\tau\left(1+p_{i}\right) x_{i}^{2}-2 p_{i} x_{i}, i=2, \ldots n .
$$

Let 
Analysis of a Congestion Model in a Wireless Access Network with One Bottleneck Router and N TCP Flows

$$
a_{i}^{2}=\frac{1}{\tau\left(1+p_{i}\right)}, i=1, \ldots n, \quad c_{i}^{2}=\frac{p_{1}^{2}}{\tau\left(1+p_{1}\right)}-\frac{p_{i}^{2}}{\tau\left(1+p_{i}\right)}, i=2, \ldots n,
$$

and

$$
X_{i}: x_{i}-p_{i} a_{i}^{2}, i=1, \ldots, n
$$

From (13) we have:

$$
\frac{X_{1}^{2}}{a_{1}^{2} c_{1}^{2}}-\frac{X_{i 1}^{2}}{a_{i}^{2} c_{i}^{2}}-1=0, i=2, \ldots, n
$$

For $i=2$, from (16) we found that $X_{1}(\alpha)$ given by:

$$
X_{1}(\alpha)=a_{1} c_{2} \cosh (\alpha), X_{1}(\alpha)=a_{2} c_{2} \sinh (\alpha)
$$

is a solution of (16), for $\alpha \in R$.

For (17) and (15) we have:

$$
\begin{aligned}
& x_{1}(\alpha)=a_{1} c_{2} \cosh (\alpha)+p_{1} a_{1}^{2}, \\
& x_{2}(\alpha)=a_{2} c_{2} \sinh (\alpha)+p_{1} a_{2}^{2}, \\
& x_{i}(\alpha)=a_{i} \sqrt{c_{2}^{2} \cosh ^{2}(\alpha)-c_{i}^{2}}+p_{i} a_{i}^{2}, i=3, \ldots, n .
\end{aligned}
$$

We determine $\alpha \in R$ so that, relations (18) satisfy the equation:

$$
f(\alpha)=F\left(\sum_{i=1}^{n} x_{i}(\alpha)\right)-c=0 .
$$

If $f(0)=F\left(\sum_{i=1}^{n} x_{i}(\alpha)\right)-c<0$ then there is $\alpha_{0}>0$ so that $f\left(\alpha_{0}\right)=0$.

Proposition 1: The equilibrium point $\left(x_{1}^{*}, x_{2}^{*}, \ldots, x_{n}^{*}, q^{*}\right)$ of the system (6) is given by: 
Gabriela Mircea, Mihaela Muntean, Mihaela Neamtu

$$
\begin{aligned}
x_{1}^{*} & =x_{1}\left(\alpha_{0}\right), x_{2}^{*}=x_{2}\left(\alpha_{0}\right), \ldots, x_{n}^{*}=x_{n}\left(\alpha_{0}\right) \\
q^{*} & =\frac{2}{2\left(k\left(2+\left(1+p_{1}\right) \tau^{2} x_{1}\left(\alpha_{2}\right)^{2}-2 p_{1} \tau x_{1}\left(\alpha_{0}\right)\right)\right)} .
\end{aligned}
$$

Consider the transformation $y_{i}(t)=x_{i}(t)-x_{i}^{*}, i=1, \ldots, n, y_{n+1}(t)=q(t)-q^{*}$.

Linearizing system (6) around the equilibrium point, we obtain:

$$
\begin{aligned}
& \dot{y}_{i}(t)=a_{i i} y_{i}(t)+a_{i, n+1} y_{n+1}(t), \\
& \dot{y}_{n+1}(t)=b \sum_{i=1}^{n} y_{i}(t-\tau),
\end{aligned}
$$

where

$$
\begin{aligned}
& a_{i i}=x_{i}^{*}\left[-\frac{1-k q^{*}}{\tau^{2} x_{i}}-\frac{k q^{*}}{2}-\frac{k p_{i} q^{*}}{2}\right], i=1, \ldots n \\
& a_{i, n+1}=x_{i}^{*}\left[-\frac{k}{\tau^{2} x_{i}}-\frac{k x_{i}^{*}}{2}-\frac{k p_{i} x_{i}^{*}}{2}+\frac{k p_{i}}{\tau}\right], i=1, \ldots n \\
& b=F^{\prime}\left(\sum_{i=1}^{n} x_{i}^{*}\right) .
\end{aligned}
$$

The characteristic equation of (22) is:

$$
D(\lambda, \tau)=P_{n+1}(\lambda, \tau)+Q_{n-1}(\lambda, \tau) e^{-\lambda \tau}
$$

where

$$
\begin{aligned}
& P_{n+1}(\lambda, \tau)=\lambda\left(\lambda-a_{11}\right)\left(\lambda-a_{22}\right) \ldots\left(\lambda-a_{n n}\right) \\
& Q_{n-1}(\lambda, \tau)=b a_{1, n+1}\left(\lambda-a_{22}\right) \ldots\left(\lambda-a_{n n}\right)+ \\
& +b a_{2, n+1}\left(\lambda-a_{11}\right)\left(\lambda-a_{33}\right) \ldots\left(\lambda-a_{n n}\right)+\ldots+ \\
& +b a_{n 1, n+1}\left(\lambda-a_{11}\right) \ldots\left(\lambda-a_{n-1, n}-1\right) .
\end{aligned}
$$

and $F^{\prime}\left(\sum_{i=1}^{n} x_{i}^{*}\right)=b$. 
Analysis of a Congestion Model in a Wireless Access Network with One Bottleneck Router and N TCP Flows

The coefficients $a_{i i}, a_{i, n+1}$ and $b$ depend on the time delay $\tau$.

For the analysis with $n=2$ we apply the geometric criterion of Kuang ([1]).

\section{Hopf bifurcation analysis of system (6) for $n=2$}

For the case $n=2$, system (6) is given by:

$$
\begin{aligned}
& \dot{x}_{1}(t)=x_{1}(t-\tau)\left[\frac{1-k q(t)}{\tau^{2} x_{1}(t)}-\frac{1}{2} k\left(1+p_{1}\right) x_{1}(t) q(t)+\frac{k}{\tau} p_{1} q(t)\right] \\
& \dot{x}_{2}(t)=x_{2}(t-\tau)\left[\frac{1-k q(t)}{\tau^{2} x_{2}(t)}-\frac{1}{2} k\left(1+p_{2}\right) x_{2}(t) q(t)+\frac{k}{\tau} p_{2} q(t)\right] \\
& \dot{q}(t)=x_{1}(t-\tau)+x_{2}(t-\tau)-c
\end{aligned}
$$

with $p_{1,} p_{2} \in[0,1], \quad p_{1} \neq p_{2}$.

Let $\left(x_{1}^{*}, x_{2}^{*}, q^{*}\right)$ the equilibrium point of system (25) given by (20), for $i=1,2$. We use the transformation

$$
y_{1}(t)=x_{1}(t)-x_{1}^{*}, y_{2}(t)=x_{2}(t)-x_{2}^{*}, y_{3}(t)=q(t)-q^{*}
$$

to linearize system (25) around $\left(x_{1}^{*}, x_{2}^{*}, q^{*}\right)$ and obtain:

$$
\begin{aligned}
& \dot{y}_{1}(t)=a_{11} y_{1}(t)+a_{13} y_{3}(t), \\
& \dot{y}_{2}(t)=a_{22} y_{2}(t)+a_{23} y_{3}(t), \\
& \dot{y}_{3}(t)=b y_{1}(t-\tau)+b \quad y_{2}(t-\tau),
\end{aligned}
$$

where

$$
\begin{aligned}
& a_{11}=x_{1}^{*}\left(\frac{-1+k q^{*}}{\tau^{2}\left(x_{1}^{*}\right)^{2}}-\frac{1}{2} k\left(1+p_{1}\right) q^{*}\right), \\
& a_{13}=x_{1}^{*}\left(-\frac{k}{\tau^{2}\left(x_{1}^{*}\right)^{2}}-\frac{1}{2} k\left(1+p_{1}\right) x_{1}^{*}+\frac{k}{\tau} p_{1}\right),
\end{aligned}
$$


Gabriela Mircea, Mihaela Muntean, Mihaela Neamtu

$$
\begin{aligned}
& a_{22}=x_{2}^{*}\left(-\frac{1-k q^{*}}{\tau^{2}\left(x_{2}^{*}\right)^{2}}-\frac{1}{2} k\left(1+p_{2}\right) q^{*}\right), \\
& a_{23}=x_{2}^{*}\left(-\frac{k}{\tau^{2}\left(x_{2}^{*}\right)^{2}}-\frac{1}{2} k\left(1+p_{2}\right) x_{2}^{*}+\frac{k}{\tau} p_{2}\right), \\
& b=F^{\prime}\left(x_{1}^{*}+x_{2}^{*}\right) .
\end{aligned}
$$

The characteristic equation associated to (26) is:

$$
D(\lambda, \tau)=\lambda^{3}-\left(a_{11}+a_{22}\right) \lambda^{2}+a_{11} a_{22} \lambda-\left(\left(a_{13}+a_{23}\right) \lambda-a_{13} a_{22}-a_{11} a_{23}\right) e^{-\lambda \tau}=0
$$

We can notice that the coefficients $a_{11}, a_{22}$ depend on $\tau$. We rewrite the characteristic equation $D(\lambda, \tau)=0$ as:

$$
D(\lambda, \tau)=P_{3}(\lambda, \tau)+Q_{1}(\lambda, \tau) e^{-\lambda \tau}=0
$$

where

$$
\begin{aligned}
& P_{3}(\lambda, \tau)=\lambda^{3}-\left(a_{11}+a_{22}\right) \lambda^{2}+a_{11} a_{22} \lambda, \\
& Q_{1}(\lambda, \tau)=b\left(a_{13}+a_{23}\right) \lambda+b\left(a_{13} a_{23}+a_{11} a_{23}\right) .
\end{aligned}
$$

Proposition 2:([1]) If $\tau>\tau_{1}$, then the following statement hold:

(i) $P_{3}(0, \tau)+Q_{1}(0, \tau) \neq 0$ and $P_{3}(i \omega, \tau)+Q_{1}(i \omega, \tau) \neq 0$ for any real positive $\omega$;

(ii) $\lim \sup \left\{\left|\frac{Q_{1}(\lambda, \tau)}{P_{3}(\lambda, \tau)}\right|:|\lambda| \rightarrow \infty, \operatorname{Re}(\lambda) \geq 0\right\}<1$;

(iii) For each $\tau$, the function $H(\omega, \tau)=\left|P_{3}(i \omega, \tau)\right|^{2}-\left|Q_{1}(i \omega, \tau)\right|^{2}$ has at most a finite number of real zeros and if there is a positive root $\omega(\tau)$ of $H(\omega, \tau)=0$ then it is continuous and differentiable in $\tau$.

Proof: A straightforward calculation leads to:

$$
P_{3}(0, \tau)+Q_{1}(0, \tau)=b\left(a_{13} a_{23}+a_{11} a_{23} \neq 0, \tau \in\left[\tau_{1}, \infty\right)\right.
$$

and

$$
\begin{aligned}
& P_{3}(i \omega, \tau)+Q_{1}(i \omega, \tau)=\left(a_{11}+a_{22}\right) \omega^{2}+b\left(a_{13} a_{23}+a_{11} a_{23}\right)+ \\
& +i\left(-\omega^{3}+\left(a_{22} a_{11}-b\left(a_{13}-a_{23}\right)\right)\right) \omega \neq 0,
\end{aligned}
$$


Analysis of a Congestion Model in a Wireless Access Network with One Bottleneck Router and N TCP Flows

then (i) holds.

\section{Using}

$$
\lim _{|\lambda| \rightarrow \infty}\left|\frac{Q_{1}(\lambda, \tau)}{P_{3}(\lambda, \tau)}\right|=\lim _{|\lambda| \rightarrow \infty}\left|\frac{-b\left(a_{13}+a_{23} \lambda+b\left(a_{13} a_{22}+a_{11} a_{23}\right)\right.}{\lambda^{3}-\left(a_{11}+a_{22}\right) \lambda^{2}+a_{11} a_{22} \lambda}\right|=0
$$

we obtain (ii). For each $\tau$, the following form of function $H(\omega, \tau)$

$$
\begin{aligned}
& H(\omega, \tau)=\omega^{6}+\left(\left(a_{11}+a_{22}\right)^{2}-2 a_{11} a_{22}\right) \omega^{4}+\left(a_{11}^{2} a_{22}^{2}-\left(a_{13}+a_{23}\right)^{2}\right) \omega^{2}- \\
& -b\left(a_{13} a_{22}+a_{11} a_{23}\right)^{2}
\end{aligned}
$$

leads to (iii).

As $\lambda=i \omega$ verifies (28) then we have:

$$
\begin{aligned}
& \sin (\omega \tau)=\frac{\omega^{3}\left(a_{11}+a_{22}\right)\left(a_{13}+a_{23}\right)-\left(\omega a_{11} a_{22}-\omega^{3}\right)\left(a_{13} a_{22}+a_{11} a_{23}\right)}{b\left(\omega^{2}\left(a_{13}+a_{22}\right)^{2}+\left(a_{13} a_{22}+a_{11} a_{23}\right)^{2}\right)}, \\
& \cos (\omega \tau)=\frac{-\omega\left(\omega a_{11} a_{22}-\omega^{3}\right)\left(a_{13}+a_{23}\right)-\omega^{2}\left(a_{11}+a_{22}\right)\left(a_{13} a_{22}+a_{11} a_{23}\right)}{b\left(\omega^{2}\left(a_{13} a_{22}\right)^{2}+\left(a_{13} a_{22}+a_{11} a_{23}\right)^{2}\right)} .
\end{aligned}
$$

Therefore,

$$
\omega^{6}+\left(a_{11}^{2}+a_{22}^{2}\right) \omega^{4}+\left(a_{11}^{2} a_{22}^{2}-b^{2}\left(a_{13}+a_{23}\right)^{2}\right) \omega^{2}-b^{2}\left(a_{13} a_{22}+a_{11} a_{23}\right)^{2}=0 .
$$

We use the notation $z=\omega^{2}$ and (31) becomes:

$$
z^{3}+r_{1} z^{2}+r_{2} z+r_{3}=0
$$

where

$$
r_{1}=a_{11}^{2}+a_{22}^{2}, r_{2}=a_{11}^{2} a_{22}^{2}-b^{2}\left(a_{13}+a_{23}\right)^{2}, r_{3}=-b^{2}\left(a_{13} a_{22}+a_{11} a_{23}\right)^{2}
$$

Due to the fact that $r_{3}<0$, the equation $h(z)=0$ has at the least one positive root, where 
Gabriela Mircea, Mihaela Muntean, Mihaela Neamtu

$$
h(z)=z^{3}+r_{1} z^{2}+r_{2} z+r_{3}
$$

Now, we use the following maps:

$$
S_{n}(\tau)=\tau-\frac{\theta(\tau)+2 n \pi}{\omega(\tau)}, \quad n \in N,
$$

where for $\tau \in\left[\tau_{1}, \infty\right)$ and $\theta(\tau) \in(0,2 \pi)$ are define by:

$$
\begin{aligned}
& \sin \theta(\tau)=\frac{-\omega\left(\left(a_{11} a_{22}-\omega^{2}\right)\left(a_{13} a_{22}+a_{11} a_{23}\right)+\omega^{3}\left(a_{11}+a_{22}\right)\left(a_{13}+a_{23}\right)\right.}{\omega^{2}\left(a_{13+} a_{23}\right)^{2}+\left(a_{13} a_{22}+a_{11} a_{23}\right)^{2}}, \\
& \cos \theta(\tau)=\frac{-\omega^{2}\left(\left(\omega a_{11} a_{22}-\omega^{2}\right)\left(a_{13}+a_{23}\right)-\left(a_{11}+a_{22}\right)\left(a_{13} a_{22}+a_{11} a_{23}\right) \omega^{2}\right.}{b \omega^{2}\left(a_{13+} a_{23}\right)^{2}+b\left(a_{13} a_{22}+a_{11} a_{23}\right)^{2}} .
\end{aligned}
$$

Equation (28) admits the roots $\lambda= \pm \omega\left(\tau_{0}\right) i, \tau_{0} \in\left(0, \tau_{1}\right)$ if and only if $S_{0}\left(\tau_{0}\right)=0$, for some $n \in N$. From [1], this pair of roots crosses the imaginary axis if $\delta\left(\tau_{0}\right)>0$ and crosses the imaginary axis from right to left if $\delta\left(\tau_{0}\right)<0$, where:

$$
\delta\left(\tau_{0}\right)=\operatorname{sign}\left\{\left.\frac{d(\operatorname{Re}(\lambda))}{d \tau}\right|_{\lambda=i \omega\left(\tau_{0}\right)}\right\}=\operatorname{sign}\left\{\left.\frac{d S_{n}(\tau)}{d \tau}\right|_{\tau=\tau_{0}}\right\}
$$

In what follows we have analyzed the stability of the system (25), when $\tau=\frac{\tau_{0}}{2}$ by discussing the stability of the following auxiliary system:

$$
\begin{aligned}
& \dot{y}_{1}(t)=c_{11} y_{1}(t)+c_{13} y_{3}(t) \\
& \dot{y}_{2}(t)=c_{22} y_{21}(t)+c_{213} y_{3}(t) \\
& \dot{y}_{3}(t)=d y_{1}(t-r)+d y_{1}(t-r),
\end{aligned}
$$

where $r \geq 0$ and

$$
\begin{aligned}
& c_{11}=\left.a_{11}\right|_{\tau=\frac{\tau_{0}}{2}}, c_{13}=\left.a_{13}\right|_{\tau=\frac{\tau_{0}}{2}}, \\
& c_{22}=\left.a_{22}\right|_{\tau=\frac{\tau_{0}}{2}}, c_{23}=\left.c_{23}\right|_{\tau=\frac{\tau_{0}}{2}} . \\
& d=\left.b\right|_{\tau=\frac{\tau_{0}}{2}} .
\end{aligned}
$$


Analysis of a Congestion Model in a Wireless Access Network with One Bottleneck Router and N TCP Flows

Then the characteristic equation of (27) is given by:

$$
\lambda^{3}-\left(c_{11}+c_{22}\right) \lambda^{2}+c_{11} c_{22} \lambda-d\left(\left(c_{13}+c_{23}\right) \lambda-c_{13} c_{22}-c_{13} c_{23}\right) e^{-\lambda r}=0 .
$$

when $r=0,(33)$ becomes:

$$
\lambda^{3}+a_{1} \lambda^{2}+a_{2} \lambda+a_{3}=0
$$

where

$$
a_{1}=-\left(c_{11}+c_{22}\right), a_{2}=c_{11} c_{22}-d\left(c_{13}+c_{23}\right), a_{3}=d\left(c_{13} c_{22}+c_{11} c_{23}\right) .
$$

Using Routh-Hurwitz stability criterion for (34), it follows:

Proposition 3: If the condition $a_{1}>0, a_{3}>0, a_{1} a_{2}>a_{3}$ is satisfied, the equilibrium $(0,0,0)$ of system (32) is locally asymptotically stable.

Consider $\lambda= \pm i \omega_{r_{0}}$, where $\omega_{r_{0}}>0$, as a simple root for equation (33). This lead to:

$$
\begin{aligned}
& d a_{4} \cos \left(r \omega_{r_{0}}\right)-d a_{3} \omega_{r_{0}} \sin \left(r \omega_{r_{0}}\right)=a_{1} \omega_{r_{0}}^{2} \\
& d a_{3} \cos \left(r \omega_{r_{0}}\right)+d a_{4} \sin \left(r \omega_{r_{0}}\right)=-\omega_{r_{0}}^{3}+a_{21} \omega_{r_{0}}
\end{aligned}
$$

From (35) we obtain:

$$
\begin{aligned}
& \sin \left(\omega_{r_{0}} r\right)=\frac{a_{4} \omega_{r_{0}}\left(\omega_{r_{0}}^{2}-a_{2}\right)-a_{1} a_{3} \omega_{r_{0}}^{3}}{d\left(a_{4}^{2}+a_{3}^{2} \omega_{r_{0}}^{2}\right)}, \\
& \cos \left(\omega_{r_{0}} r\right)=-\frac{a_{1} a_{4} \omega_{r_{0}}^{2}-a_{3} \omega_{r_{0}}\left(\omega_{r_{0}}^{3}-a_{2} \omega_{r_{0}}\right)}{d\left(a_{4}^{2}+a_{3}^{2} \omega_{r_{0}}^{2}\right)} .
\end{aligned}
$$

There four:

$$
S_{0} \omega_{r_{0}}^{8}+S_{1} \omega_{r_{0}}^{6}+S_{2} \omega_{r_{0}}^{4}+S_{3} \omega_{r_{0}}^{2}+S_{4}=0
$$

where 
Gabriela Mircea, Mihaela Muntean, Mihaela Neamtu

$$
\begin{aligned}
& S_{0}=a_{3}^{2}, \\
& S_{1}=\left(a_{4}-a_{1} a_{3}\right)^{2}-2 a_{3}\left(a_{2} a_{3}+a_{1} a_{4}\right), \\
& S_{2}=\left(a_{2} a_{3}+a_{1} a_{4}\right)^{2}-2 a_{2} a_{4}\left(a_{4}-a_{1} a_{3}\right)-d^{2} a_{3}^{4}, \\
& S_{3}=a_{2}^{2} a_{4}^{2}-2 d a_{3}^{2} a_{4}^{2}, \\
& S_{4}=-d a_{4}^{4} .
\end{aligned}
$$

Equation (36) can be rewritten as:

$$
\omega_{r_{0}}^{8}+R_{1} \omega_{r_{0}}^{6}+R_{2} \omega_{r_{0}}^{4}+R_{3} \omega_{r_{0}}^{2}+R_{4}=0
$$

where

$$
R_{1}=\frac{S_{1}}{S_{0}}, R_{2}=\frac{R_{2}}{S_{0}}, R_{3}=\frac{S_{3}}{S_{0}}, R_{4}=\frac{S_{4}}{S_{0}} .
$$

If we denote $z=\omega_{r_{0}}^{2}$ then (37), becomes:

$$
z^{4}+R_{1} z^{3}+R_{2} z^{2}+R_{3} z+R_{4}=0
$$

Equation (37) has at the least one positive root, because $\lim _{z \rightarrow \infty} h_{0}(z)=\infty$, and $R_{4}<0$, where

$$
h_{0}(z)=z^{4}+R_{1} z^{3}+R_{2} z^{2}+R_{3} z+R_{4} .
$$

Let $z_{0}$ be the positive root of the equation (40). Therefore:

$$
r(s)=\frac{1}{\omega_{r_{0}}}\left[\arccos \frac{a_{1} a_{4} \omega_{r_{0}}^{2}-a_{3} \omega_{r_{0}}^{2}\left(\omega_{r_{0}}^{2}-a_{2}\right)}{d\left(a_{3}^{2}+a_{3}^{2} \omega_{r_{0}}^{2}\right)}+2 s \pi\right],
$$

where $s=0,1,2, \ldots$ and $\omega_{r_{0}}=\sqrt{z_{0}}$.

Now, we consider $\mu(r)=\mu(r)+i \omega_{r}(r)$, a root of (33) with $\mu\left(r_{0}\right)=0$, $\omega_{r}\left(r_{0}\right)=\omega_{r_{0}}$. Differentiating equation (33) with respect to $r$, we get: 
Analysis of a Congestion Model in a Wireless Access Network with One Bottleneck Router and N TCP Flows

$$
\frac{d \lambda(r)}{d r}=\frac{-\lambda e^{-\lambda r}\left(C_{2}-C_{4}\right)}{C_{1}+C_{2} e^{-\lambda r}+\left(C_{3} \lambda+C_{4}\right) r e^{-\lambda r}},
$$

where

$$
\begin{aligned}
& C_{1}=3 \lambda^{2}-\left(c_{11}+c_{22}\right) \lambda+c_{11} c_{22}, \\
& C_{2}=-d\left(c_{13}+c_{23}\right), \\
& C_{3}=d\left(c_{13}+c_{23}\right) \lambda, \\
& C_{4}=-d\left(c_{13} c_{22}+c_{11} c_{23}\right) .
\end{aligned}
$$

For $\lambda\left(r_{0}\right)=i \omega_{r_{0}}$, from (42), we get:

$$
\left.\operatorname{Re}\left(\frac{d \lambda(r)}{d r}\right)^{-1}\right|_{\lambda=\lambda\left(r_{0}\right)}=\frac{A_{1} A_{2}-B_{1} B_{2}}{A_{2}^{2}+B_{2}^{2}}
$$

where

$$
\begin{aligned}
& A_{1}=2 \omega_{r_{0}}\left(c_{11}+c_{22}\right) \sin \left(\omega_{r_{0}} r_{0}\right)+\left(2 c_{11} c_{22}-3 \omega_{r_{0}}^{2}\right) \cos \left(\omega_{r_{0}} r_{0}\right), \\
& A_{2}=d\left(c_{13}+c_{23}\right) \omega_{r_{0}}^{2}, \\
& B_{1}=c_{11} c_{22} \sin \left(\omega_{r_{0}}\right), \\
& B_{2}=d\left(c_{13} c_{23}+c_{11} c_{23}\right) \omega_{r_{0}} .
\end{aligned}
$$

From the above discussion, the transversality condition holds:

$$
\left.\operatorname{Re}\left(\frac{d \lambda(r)}{d r}\right)^{-1}\right|_{\lambda=\lambda\left(r_{0}\right)} \neq 0 .
$$

Theorem 4: The equilibrium point of the linearized system (32) is locally asymptotically stable when $r<r_{0}$. If $r_{0}\left(\tau_{0}\right)>\frac{\tau_{0}}{2}$, then the equilibrium point of the wired access network with one bottleneck router and two TCP flow described by (25) is locally asymptotically stable when $\tau=\frac{\tau_{0}}{2}$.

According to [2] we have:

DOI: $10.24818 / 18423264 / 52.3 .18 .01$ 
Proposition 5: If the function $S_{0}(\tau)$ has positive zeros in $\left(0, \tau_{1}\right)$, the equilibrium point $\left(x_{1}^{*}, x_{2}^{*}, q^{*}\right)$ of system $(25)$ is asymptotically stable for all $\tau \in\left(\tau_{1}, \tau_{0}\right)$ and becomes unstable for staying in some right neighborhood of $\tau_{0}$. Therefore, system (25) undergoes Hopf bifurcation when $\tau<\tau_{0}$.

\section{Numerical Simulation}

Numerical simulations are done with Maple, Matlab and the following parameters: $c=5, \quad p_{1}=0.8, p_{2}=0.2, \quad k=0.001, \tau_{0}=2.06$. The equilibrium point of system (25) is $x_{1}^{*}=2.33, x_{2}^{*}=2.6645, q^{*}=55.615$. From the above findings, the equilibrium point $\left(x_{1}^{*}, x_{2}^{*}, q^{*}\right)$ is asymptotically stable when $\tau<\tau_{0}$ (see Figure 1).

When $\tau$ takes the value $\tau_{0}=2.06,\left(x_{1}^{*}, x_{2}^{*}, q^{*}\right)$ loses its stability and a Hopf bifurcation takes place (Figure 2).

We can conclude that the numerical example verifies the theoretical findings.

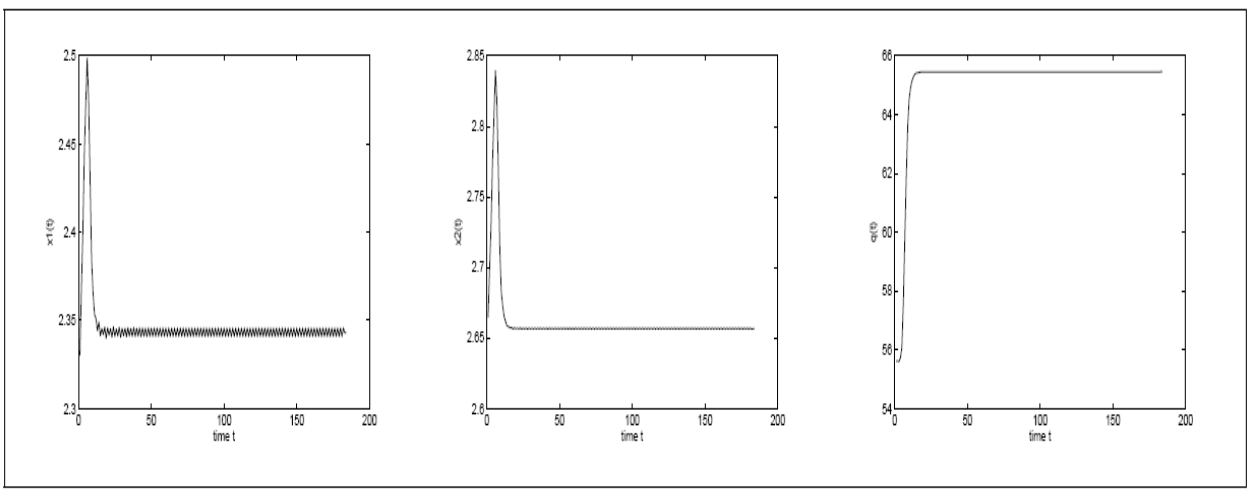

Figure 1: The orbits $\left(t, x_{1}(t)\right),\left(t, x_{2}(t)\right)$, and $(t, q(t))$ when the round trip time is $\mathbf{1 . 9}$ 
Analysis of a Congestion Model in a Wireless Access Network with One Bottleneck Router and N TCP Flows

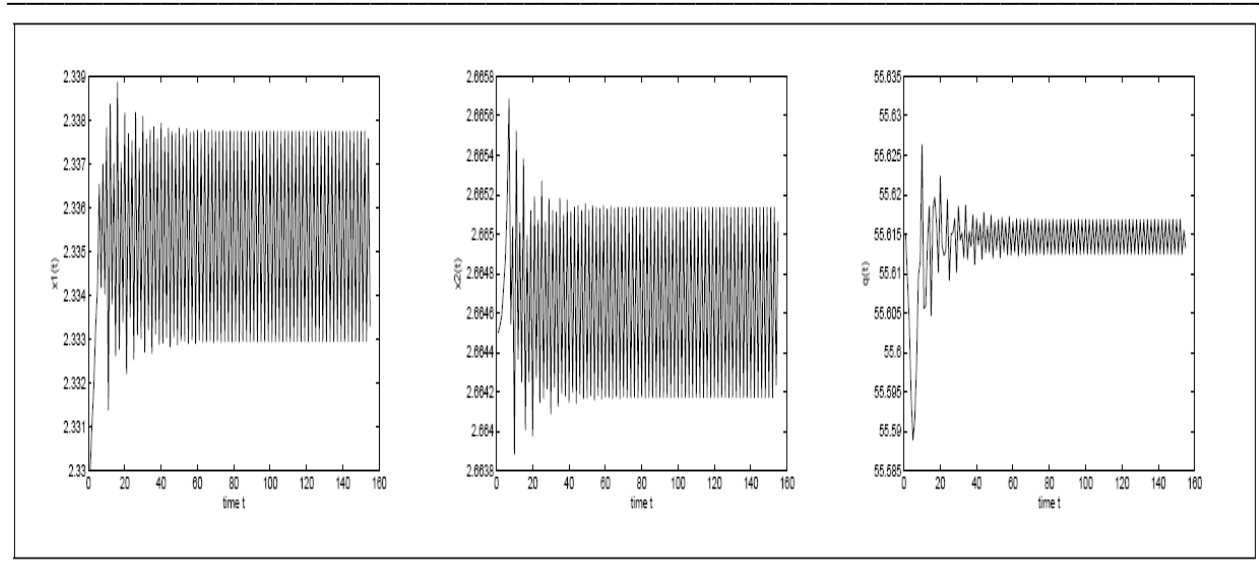

Figure 2: The orbits $\left(t, x_{1}(t)\right),\left(t, x_{2}(t)\right)$, and $(t, q(t))$ when the round trip time is 2.06

\section{Conclusion and future works}

This paper deals with a congestion model in a wireless access network with one bottleneck router and $n \geq 2$ TCP flows. When two different TCP flows pass through the router, a nonlinear dynamical system with three differential equations describes the model. Two equal time delays that stand for round trip times of flows, are introduced.

The equilibrium point is determined and we provide sufficient conditions for its stability by analyzing the characteristic equation associated to the linearized system. The round trip time is considered as bifurcation parameter and when it takes a critical value we proved the existence of the Hopf bifurcation. A family of periodic orbits bifurcates from the equilibrium point. We used Maple and Matlab for the numerical simulations to verify the theoretical things.

Due to the fact that there are perturbations in our future work we will take into consideration the stochastic model with distributed time delay.

\section{ACKNOWLEGEMENT}

This work was conducted by the authors as associate members of ECREBEast European Center for Research in Economics and Business, Faculty of Economics and Business Administration, West University of Timisoara (http://ecreb.ro). 
Gabriela Mircea, Mihaela Muntean, Mihaela Neamtu

\section{REFERENCES}

[1] Beretta, E., Kuang, Y.(2002), Geometric Stability Switch Criteria in Delay Differential Systems with Delay-dependent Parameters; SIAM J. Math. Anal., vol. 33, no. 5, pp. 1144-1165;

[2] Ding, D.W., Qin, X.M., Wangetal, N. (2013), Hybrid Control of Hopf Bifurcation in a Dual Model of Internet Congestion Control System; Nonlinear Dynamics, pp.110;

[3] Dong, T., Liao, X., Huang, T. (2013), Dynamics of a Congestion Control Model in a Wireless Access Network; Nonlinear Analysis: Real World Applications, 14, pp. 671683;

[4] Gentile, F.S., Moiola, J.L., Paolini, E.E., (2014), Nonlinear Dynamics of Internet Congestion Control: A Frequency-domain Approach; Communications in Nonlinear Science and Numerical Simulation, vol.19, no.4, pp.11131127;

[5] Guo, S., Liao, X., Li, C. (2008), Stability and Hopf Bifurcation Analysis in a Novel Congestion Control Model with Communication Delay; Nonlinear Analysis: Real World Applications, vol. 9, no. 4, pp. 1292-1309;

[6] Hollot, C.V., Misra, V., Towsley, D., Gong, W. (2002), Analysis and Design of Controllers for AQM Routers Supporting TCP Flows; IEEE Transactions on Automatic Control, vol.47, no.6, pp.945-959;

[7] Mircea, G. (2008), Internet Congestion Control Model; Proceedings of the 9th International Conference on Mathematics and Computers in Business and Economics, Bucharest, Romania, pp. 158-162;

[8] Mircea, G., Neamtu, M., Opris, D. (2011), Deterministic and Stochastic Internet-style Networks with Delay; Recent Advances in Applied \& Biomedical Informatics and Computational Engineering in Systems Applications, pp. 239-244;

[9] Mircea, G., Neamtu, M., Ciurdariu, A, Opris, D. (2009), Numerical Simulations for Dynamic Stochastic and Hybrid Models of Internet Networks; Transactions on Mathematics, Vol.8, Issue 12, pp. 679-688, 2009;

[10] Mircea, G., Opris, D. (2009), Neimark-Sacker and Flip Bifurcations in a Discrete-time Dynamic System for Internet Congestion; Transaction on Mathematics, Vol.8, no. 2, pp. 63-72;

[11] Liu, F, Xiong, G., Guanetal, Z.H. (2013), Stability Analysis and Control Hopf Bifurcation in a FAST TCP Model; Proceedings of the IEEE 32nd Chinese Control Conference, pp.1076-1080;

[12] Zhao, W., Sun, X. Wang, H. (2014), Hopf Bifurcation and Stability Analysis of a Congestion Control Model with Delay in Wireless Access Network, Hindawi Publishing; Vol. 2014, Article ID: 632564, 12 pages. 